\title{
Self-Regenerating Soft Biophotovoltaic Devices
}

\author{
Xinkai Qiu, ${ }^{\dagger, \ddagger, \S}$ Olga Castañeda Ocampo, ${ }^{\dagger, \ddagger, \S}$ Hendrik W. de Vries, ${ }^{\dagger} \odot$ Maikel van Putten, ${ }^{\dagger}$

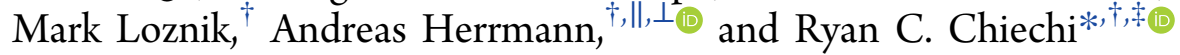 \\ ${ }^{\dagger}$ Zernike Institute for Advanced Materials, Nijenborgh 4, 9747 AG Groningen, The Netherlands \\ ${ }^{\ddagger}$ Stratingh Institute for Chemistry, University of Groningen, Nijenborgh 4, 9747 AG Groningen, The Netherlands
}

\section{Supporting Information}

\begin{abstract}
This paper describes the fabrication of soft, stretchable biophotovoltaic devices that generate photocurrent from photosystem I (PSI) complexes that are selfassembled onto $\mathrm{Au}$ electrodes with a preferred orientation. Charge is collected by the direct injection of electrons into the $\mathrm{Au}$ electrode and the transport of holes through a redox couple to liquid eutectic gallium-indium (EGaIn) electrodes that are confined to microfluidic pseudochannels by arrays of posts. The pseudochannels are defined in a single fabrication step that leverages the non-Newtonian rheology of EGaIn. This strategy is extended to the fabrication of reticulated electrodes that are inherently stretchable. A simple shadow

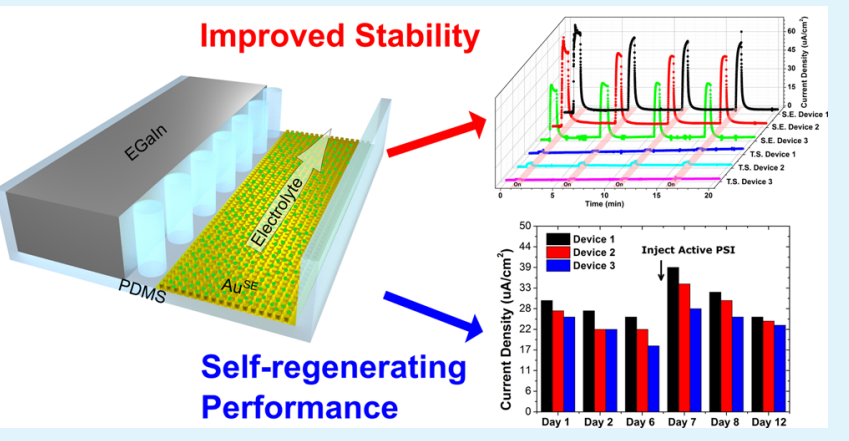
evaporation technique is used to increase the surface area of the Au electrodes by a factor of approximately $10^{6}$ compared to planar electrodes. The power conversion efficiency of the biophotovoltaic devices decreases over time, presumably as the PSI complexes denature and/or detach from the Au electrodes. However, by circulating a solution of active PSI complexes the devices self-regenerate by mass action/self-assembly. These devices leverage simple fabrication techniques to produce complex function and prove that photovoltaic devices comprising PSI can retain the ability to regenerate, one of the most important functions of photosynthetic organisms.
\end{abstract}

KEYWORDS: photosystem I, self-assembly, EGaIn, microfluidics, cofabrication, stretchable photovoltaics

\section{INTRODUCTION}

Emerging challenges in the fabrication of microelectronic devices are driving research into simple, rapid, and inexpensive fabrication strategies that are amenable for a large variety of materials and architectures. ${ }^{1,2}$ Cofabrication is such a strategy ${ }^{3}$ which simplifies the fabrication of multicomponent microelectronic devices compared to, for example, multilayer microfabrication, a commonly-used technique for fabricating field-effect transistors, photovoltaic devices, etc. It uses microfluidic channels, fabricated by using soft lithography, to provide the single structural basis (the master) for all the components and their functions that are required in the final device, which is produced by sealing the channels against a flat substrate and filling them with different materials to generate desired functions. Cofabrication is capable of generating microcomponents that are correctly aligned, in a single lithographic step, over a large surface area, avoiding registration and improving the efficiency of fabrication. It is also useful as a laboratory-scale rapid-prototyping technique because devices can be manufactured from inexpensive materials (e.g., poly(dimethylsiloxane) (PDMS), ${ }^{4}$ thiolene resins, ${ }^{5}$ polycarbonate, ${ }^{6}$ glass, ${ }^{7,8}$ etc.) and simple methods (e.g., soft lithography, heat embossing, etc.) while consuming less solvent and photoresist compared to conventional fabrication strategies.
Cofabricated microfluidic systems based on silicone elastomers such as PDMS and Ecoflex ${ }^{9}$ have shown potential as wearable electronic devices, including health monitors. ${ }^{10,11}$ However, the incorporation of conductive microcomponents with correct alignment and stable electronic properties remains a challenge. The common approach to fabricate conductive microcomponents on flat substrates involves the patterning (e.g., shadow mask and lithography) and deposition (e.g., thermal evaporation, electron beam evaporation, and sputtering) of metals. ${ }^{12-14}$ As the size and complexity of the pattern grows, aligning the microcomponents with microfluidic channels without cracking the pattern during fabrication becomes more difficult. Alternative approaches focus on installing thin metallic films onto the side walls of the microfluidic channels by deposition at an angle ${ }^{15}$ and ion milling to etch thin films into desired shapes, ${ }^{16}$ which adds complexity to the fabrication. Organic materials mitigate some of this complexity, but their relatively low electrical mobility and oxidative instability remain problematic. ${ }^{17}$ Eutectic gallium-indium (EGaIn 75\%, Ga 25\% In by weight, melting point $15.5{ }^{\circ} \mathrm{C}$ ) combines the advantageous properties of

Received: July 4, 2018

Accepted: October 8, 2018

Published: October 8, 2018 
metallic films and organic materials. It rapidly forms flexible electronic components in pre-patterned microfluidic channels by injection at room temperature, an example of cofabrication that obviates the need for registration and multistep fabrication. ${ }^{18}$ EGaIn has also been used to fabricate stretchable interconnects ${ }^{19}$ and antennas ${ }^{20}$ in microfluidic systems.

A comparable approach to simplifying the fabrication of devices with complex functionality is to co-opt naturally occurring structures such as photosystem I (PSI) to fabricate photovoltaic devices. $^{21-23}$ Combined with self-assembly, complex nanostructures can be fabricated simply, en masse. $^{24-28}$ While the efficiencies of such biophotovoltaic (bio-PV) devices remain far from commercial viability, ${ }^{29}$ cofabrication makes it possible to explore self-repair and selfregeneration, which are vital to living systems, but do not readily translate to thin-film devices. ${ }^{30-32}$ Cofabricated microfluidic channels enable the incorporation of materials in different forms that can be removed or replaced in the same device to produce microheaters, ${ }^{33}$ in-plane electromagnets, ${ }^{33,34}$ optofluidic waveguides, ${ }^{35,36}$ switches and couplers, ${ }^{36}$ lenses, ${ }^{37}$ light sources, ${ }^{38,39}$ microdroplet generators, ${ }^{40-44}$ and chemical reactors. ${ }^{45,46}$ Combining these traits with the self-assembly of PSI on metallic electrodes potentially allows the replacement of inactive PSI, in operando.

In this paper, we introduce a simple strategy to construct a stretchable network of EGaIn microelectrodes in a cofabricated microfluidic chip to form bio-PV devices that operate similarly to dye-sensitized solar cells. ${ }^{47}$ Due to the unique rheology of EGaIn, regularly-spaced PDMS posts confine the EGaIn microelectrodes to pseudochannels that are spatially separated from other fluid-filled microfluidic channels, but are still in chemical contact. ${ }^{48}$ This design (electro)chemically couples EGaIn to $\mathrm{Au}$ electrodes functionalized with PSI via an electrolytic medium. By reducing the surface free-energy of EGaIn during the injection, we significantly increased the length of the EGaIn microelectrodes, enabling the formation of reticulated electrodes/channels to increase the volume fraction of the PDMS chip taken up by the electrodes. The reticulated EGaIn electrodes are inherently stretchable and can be deformed without losing their structure or electrical conductivity. We fabricated bio-PV devices by immobilizing trimers of PSI via self-assembly with selective orientation onto nanostructured Au electrodes in microfluidic channels flanked by EGaIn microelectrodes. Circulating PSI through the resulting solar cells caused the devices to self-regenerate via the spontaneous replacement of inactive PSI by active PSI from solution.

\section{RESULTS AND DISCUSSION}

Biophotovoltaic Device Design. A typical device begins with a straight $475 \mu \mathrm{m}$-wide channel formed in PDMS and partitioned by a row of $50 \mu \mathrm{m}$-diameter posts separated by 50 $\mu \mathrm{m}$ as shown in Figure 1A. A strip of smooth, templatestripped $^{49} \mathrm{Au}\left(\mathrm{Au}^{\mathrm{TS}}\right)$ is placed on a flat PDMS substrate and modified with a self-assembled monolayer (SAM) of a short linker molecule (sodium 3-mercapto-1-propanesulfonate, MPS). As depicted in Figure 1B, when exposed to a solution of PSI, this "director" SAM biases the PSI to self-assemble (Figure S7) such that the electron transport chain will inject electrons into the $\mathrm{Au}^{\mathrm{TS}}$ electrode when illuminated. ${ }^{25}$ After functionalizing the $\mathrm{Au}^{\mathrm{TS}}$ electrode with PSI, the channel is sealed against it such that half of the channel overlaps with the electrode. To form the counter electrode, EGaIn is injected
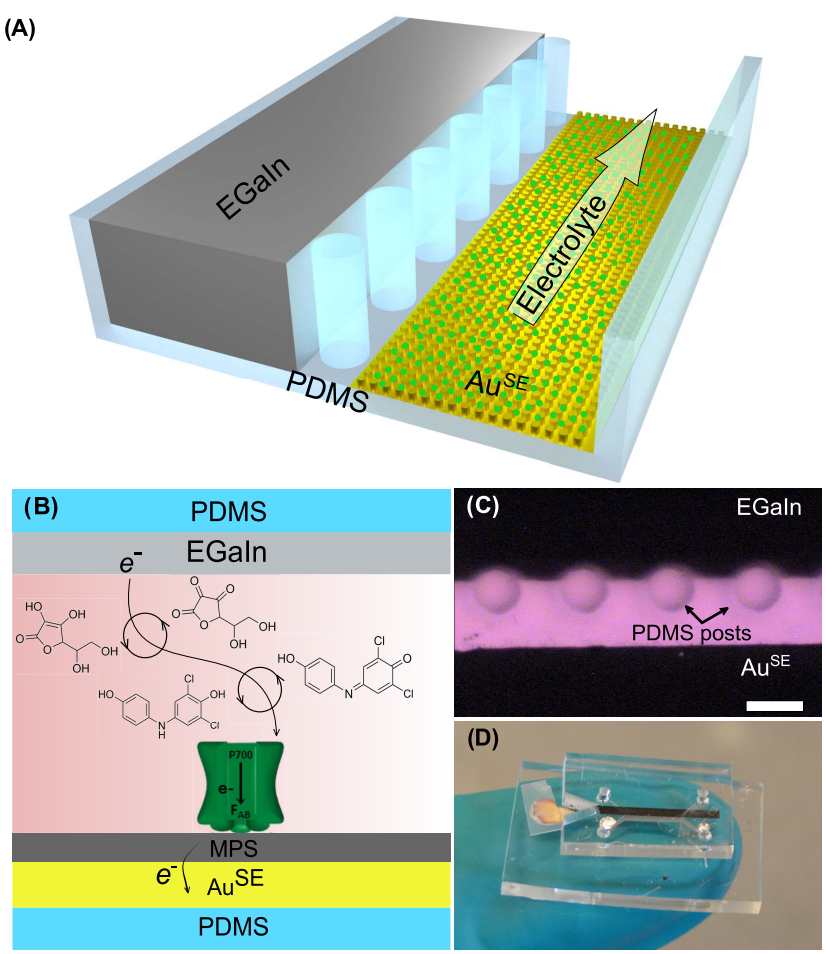

Figure 1. (A) Schematic illustration showing the cross-section of the biophotovoltaic device: the EGaIn electrode (gray) is separated from the PSI-functionalized Au electrode (yellow) by PDMS posts; PSI complexes (green) are immobilized on the surface of the $\mathrm{Au}$ electrode; the electrolyte fills the cavity above the Au electrode and contacts the EGaIn electrode. (B) Photovoltaic mechanism: PSI complexes form a self-assembled monolayer on the surface of $\mathrm{Au}$, which is functionalized with a short linker that influences the orientation of the PSI. An aqueous solution of ascorbic acid and DCPIP dissolved in the electrolyte reduces PSI after it injects a photoexcited electron, restoring its ground state. (C) The alignment of the electrodes inside microfluidic channels under an optical microscope: the PDMS posts are $50 \mu \mathrm{m}$ in diameter, the distance between two posts is $50 \mu \mathrm{m}$. The scale bar is $100 \mu \mathrm{m}$. (D) A photograph of a completed device that uses conductive silver paste to connect the Au electrode to an external load.

into the side of the channel that is not occupied by the $\mathrm{Au}^{\mathrm{TS}}$ / PSI electrode. The array of the posts both guides the flow of EGaIn into this pseudochannel and keeps it confined on one side of the posts such that it occupies the same channel as the $\mathrm{Au}^{\mathrm{TS}}$ electrode, but does not touch it, as is shown in Figure 1C. Because the $\mathrm{Au}^{\mathrm{TS}}$ electrodes are aligned with the PDMS posts by hand, the distance between the $\mathrm{Au}^{\mathrm{TS}}$ electrode and the posts ranges from 10 to $80 \mu \mathrm{m}$. To complete a bio-PV device, a redox couple consisting of an aqueous solution of ascorbic acid (5 mmol) and 2,6-dichlorophenolindophenol (DCPIP, 0.1 $\mathrm{mmol}$ ) is injected into the microfluidic channel that is defined by the EGaIn electrode such that the EGaIn and $\mathrm{Au}^{\mathrm{TS}}$ are both in contact with the solution, but do not touch each other. Figure 1D shows a completed device. We chose a redox couple that is widely used to study the activity of PSI by oxygen consumption measurement, but not necessarily optimized for photovoltaics. It operates in three steps: (i) PSI absorbs a photon and injects an electron into the $\mathrm{Au}^{\mathrm{TS}}$ electrode; (ii) DCPIP transfers an electron to PSI, regenerating its ground state; and (iii) ascorbic acid transfers an electron to DCPIP and then diffuses to the EGaIn electrode where it is reduced by EGaIn to complete the redox cycle ${ }^{50}$ (Figures $1 \mathrm{~B}$ and S13). 
As expected, illuminating the cofabricated PDMS chips containing $\mathrm{EGaIn} / \mathrm{Au}^{\mathrm{TS}} / \mathrm{PSI}$ leads to a measurable shortcircuit current density $J_{S C}$. To ensure that PSI is responsible for $J_{S C}$, we measured control devices that lack PSI or that are fabricated using boiled (denatured) PSI. These results are summarized in Figure S2 and Table S2; while devices lacking active PSI result in a small $J_{\mathrm{SC}}$, it increases dramatically for devices containing active PSI. The non-zero $J_{S C}$ from the control devices are likely due to (inefficient) charge-transfer between the redox couple and the $\mathrm{Au}$ electrode under illumination either from the excitation of DCPIP or plasmon resonances in $\mathrm{Au}$.

Electrode Surface Area. While the bio-PV device described above is functional and simple to fabricate, there are three parameters that can be optimized to increase the power conversion efficiency $\eta$ : (i) the redox couple, (ii) the volume fraction of the PDMS chip occupied by the active PV components, and (iii) the surface area of the Au electrode. We focused only on the latter two because they impact the complexity of the device.

To maximize the surface area of the Au electrode, we used a shadow evaporation technique that we developed previously. ${ }^{51}$ Briefly, $\mathrm{Au}$ is vapor-deposited at an angle onto a rotating array of porous alumina (an anodic aluminum oxide (AAO) membrane filter, Figure S8) that is later dissolved to leave behind an Au substrate decorated with densely-packed, hollow nanotubes of Au standing perpendicular to the surface (Figure S9). The thickness of the walls, diameter, and height of the tubes can be controlled by the angle, deposition time, and pore diameter, which we optimized to accommodate PSI. The surface area of the shadow-evaporated $\mathrm{Au}\left(\mathrm{Au}^{\mathrm{SE}}\right)$ electrodes increases by a factor of $\sim 10^{6}$ compared to $\mathrm{Au}^{\mathrm{TS}}$ (see the Supporting Information). To test the hypothesis that this increase in the surface area results in higher $J_{\mathrm{SC}}$, as calculated from the macroscopic area of the electrodes, we performed systematic measurements on the performance of the bio-PV devices fabricated using both. Figure 2 compares the performance of two sets of devices fabricated from template $\mathrm{Au}^{\mathrm{TS}}$ and $\mathrm{Au}^{\mathrm{SE}}$ under four cycles of light $(1 \mathrm{~min})$ and dark (4 min) over a period of $20 \mathrm{~min}$. For devices fabricated using $\mathrm{Au}^{\mathrm{SE}}, J_{\mathrm{SC}}\left(\mu \mathrm{A} / \mathrm{cm}^{2}\right)=64,64$, and 47 . For devices fabricated using $\mathrm{Au}^{\mathrm{TS}}, J_{\mathrm{SC}}\left(\mu \mathrm{A} / \mathrm{cm}^{2}\right)=2,4$, and 1 (see Table $\mathrm{S} 1$ ). The average $J_{\mathrm{SC}}$ of the devices fabricated using $\mathrm{Au}^{\mathrm{SE}}$ was a factor of

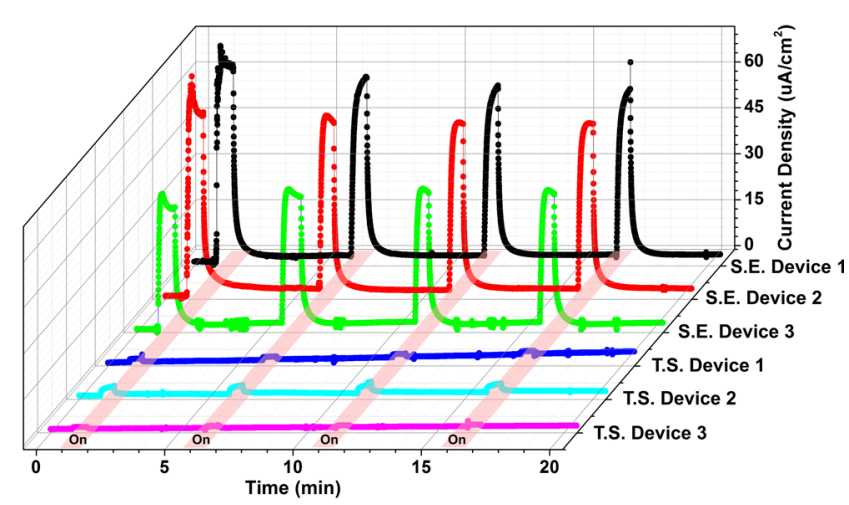

Figure 2. Comparison of $J_{\mathrm{SC}}$ between two sets of three devices fabricated using $\mathrm{Au}^{\mathrm{TS}}$ (blue, cyan, and pink) and $\mathrm{Au}^{\mathrm{SE}}$ (black, red, and green) under cycles of illumination $(1 \mathrm{~min})$ and dark $(4 \mathrm{~min})$ over a total of 20 min. Light pink bars indicate the periods in which the devices were illuminated.
75 larger than that of those fabricated using $\mathrm{Au}^{\mathrm{TS}}$, reflecting the increased density of PSI on $\mathrm{Au}^{\mathrm{SE}}$ electrodes. We observed a maximum decrease of $16 \%$ in $J_{S C}$ after the first two light/dark cycles in the devices fabricated using $\mathrm{Au}^{\mathrm{SE}}$, but it remained stable in the later cycles over more than 10 min under ambient conditions or under continuous illumination (Figure S3). Comparing the stability of PSI-based bio-PV devices to literature reports is difficult because other devices are typically only tested for about $30 \mathrm{~s}^{29,52,53}$ and use redox couples dissolved in organic solvents ${ }^{29}$ that quickly denature the PSI. Our devices are intrinsically more stable (which we discuss in more detail below) because they use aqueous electrolytes.

We further investigated the $J / V$ characteristics of bio-PV devices fabricated using $\mathrm{Au}^{\mathrm{SE}}$ to measure the power conversion efficiency $\eta$ and the open-circuit voltage $V_{\mathrm{OC}}$. Figure $3 \mathrm{~A}$ shows
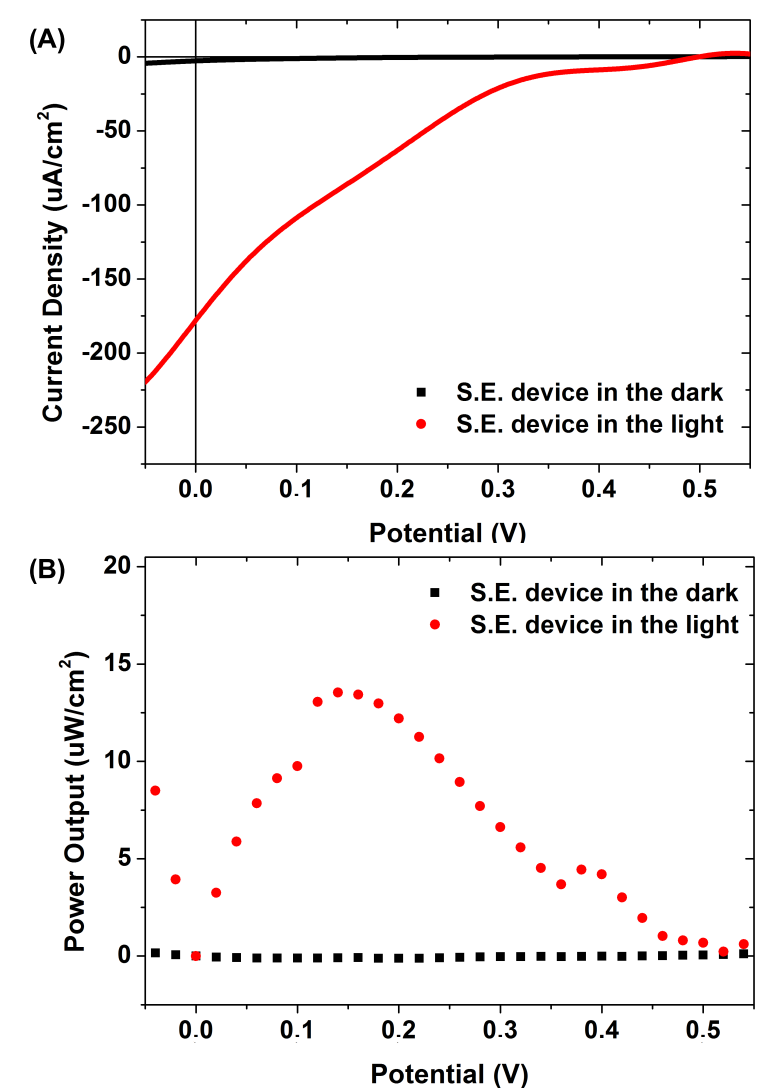

Figure 3. (A) $J / V$ curves and (B) power output curves of the device tested in the dark (black) and under the illumination of red laser (red). The power conversion efficiency of a typical device is $0.002 \%$ with a fill factor of 0.14 .

the $J / V$ curve of a typical device measured in the dark and under irradiation with $655 \mathrm{~nm}$ light; $V_{\mathrm{OC}}=0.5 \mathrm{~V}$ and $J_{\mathrm{SC}}=180$ $\mu \mathrm{A} / \mathrm{cm}^{2}$. Figure $3 \mathrm{~B}$ shows the power output as a function of potential from which we calculate a maximum power output of $13.5 \mu \mathrm{W} / \mathrm{cm}^{2}$ and a fill factor (FF) of 0.15 to give $\eta=$ $0.0025 \%$. While this $\eta$ is clearly not technologically useful, it is reasonably compared to other bio-PV devices based on $\mathrm{PSI}^{23,29}$ and, as mentioned above, our devices are intrinsically more stable, which leaves room for further optimization. For example, using phage display to increase the fraction of PSI with the correct orientation ${ }^{24}$ to inject an electron into the $\mathrm{Au}^{\mathrm{SE}}$ electrode and substituting $\mathrm{Au}$ with other materials to better match the work function to the excited state of PSI (the 

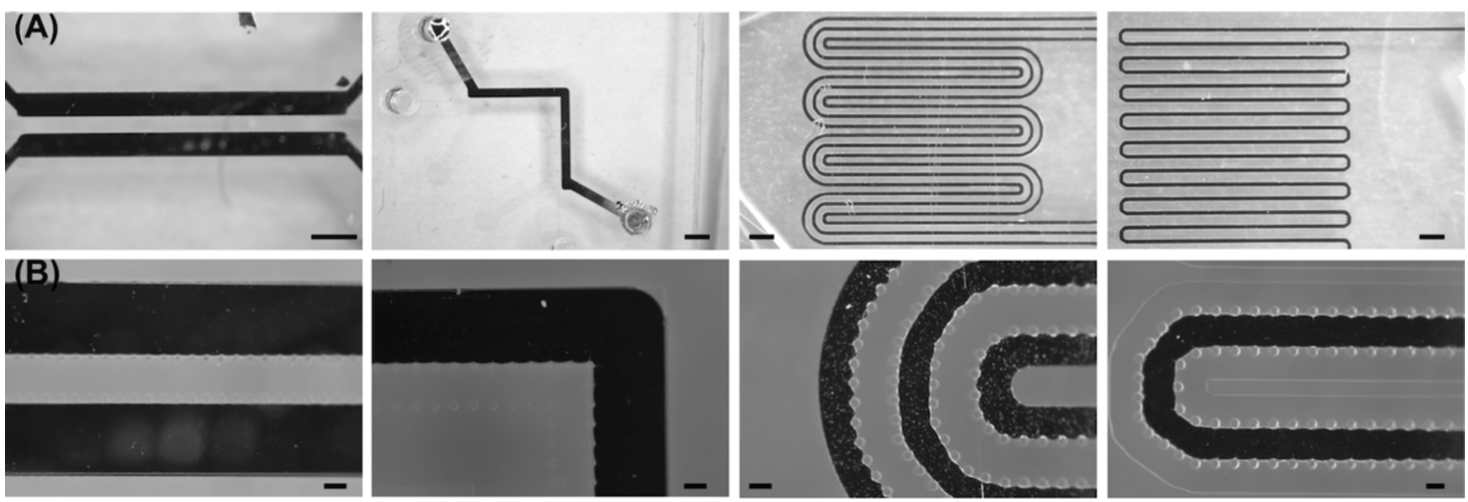

Figure 4. A) Optical micrographs of four types of inherently aligned cofabricated microfluidic channels, scale bars are $1000 \mu \mathrm{m}$. (B) Magnified views of the four structures, scale bars are $100 \mu \mathrm{m}$. EGaIn appears black in these images.
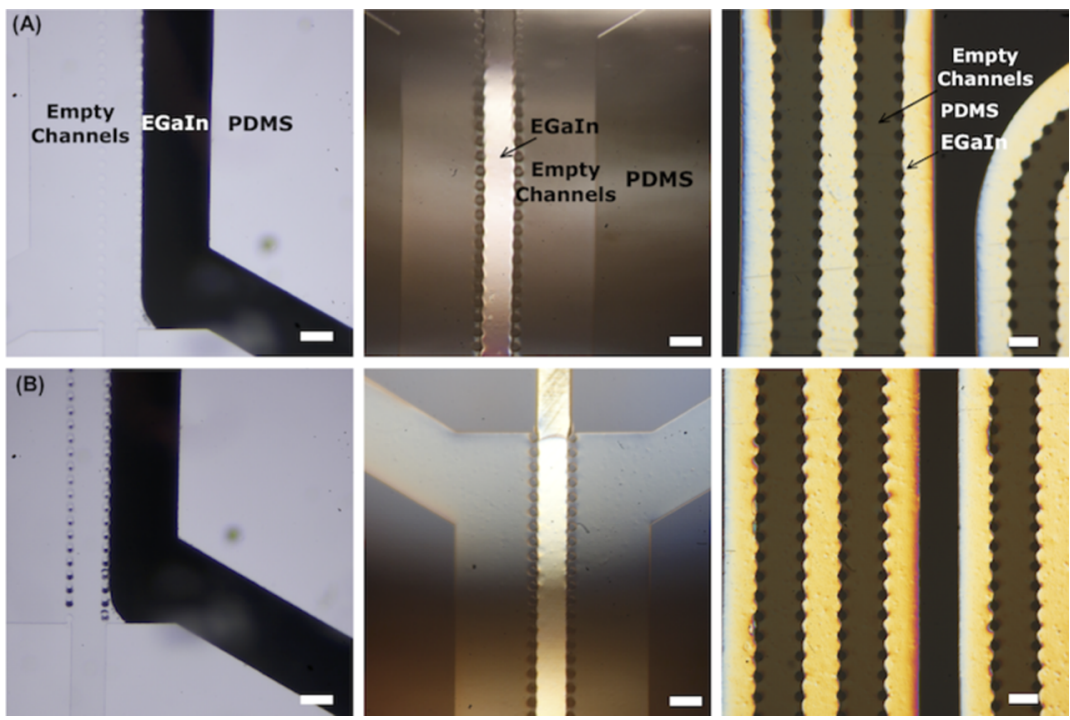

Figure 5. Three types of EGaIn microelectrodes pictured (A) before and (B) after stretching. Scale bars are $150 \mu \mathrm{m}$. EGaIn appears black in the left-most images and reflective in the others.

shadow evaporation technique is not limited to $\mathrm{Au}$ ), in addition to optimizing the redox couple. However, the purpose of this work is to demonstrate the use of cofabrication to make soft, self-regenerating devices-to focus on the unique properties of PSI rather than maximizing $\eta$, which remains as a future challenge. We also characterized the device under AM1.5 solar illumination, however, the photoresponse from the device was too weak to distinguish from the noise level. This is possibly due to the low absorption of the monolayer of PSI to incident light and significantly smaller power influx of solar illumination compared to laser light.

Reticulated Cofabricated Electrodes. Due to the design of our devices, that places the EGaIn electrode perpendicular to the $\mathrm{Au}^{\mathrm{SE}}$ electrode, the PSI complexes are illuminated through PDMS rather than, for example, a transparent electrode material and the devices can be stacked since the electrodes are completely encapsulated. This design has the advantage that PDMS is completely transparent at the wavelengths of light absorbed by PSI and the disadvantage that the volume fraction of a square chip occupied by straight channels is low enough that most of the light will pass through without interacting with PSI. Reticulated channels significantly increase the fraction of the chip occupied by $\mathrm{Au}^{\mathrm{SE}}$, but thus far only straight cofabricated channels have been demonstrated.
To investigate whether cofabricated channels can turn corners and still confine EGaIn behind an array of posts, we fabricated four types of EGaIn microelectrodes by injecting the liquid metal into PDMS microchannels: (A) two straight electrodes flanking a fluidic channel; (B) L-shape electrode; (C) three reticulated electrodes; and (D) a reticulated electrode flanked by two fluidic channels (Figure 4). EGaIn electrode A has been previously reported, but is limited to straight channels. ${ }^{48}$ We extended this fabrication technique to produce the latter three types of electrodes, which increase the volume fraction (and surface area) of EGaIn that can be incorporated in a single step. The PDMS posts not only separate channels, but also guide the flow of EGaIn during injection. We reproduced electrode (A) as reported ${ }^{48}$ where the width of the channel was $375 \mu \mathrm{m}$ and distance between two neighboring posts was $50 \mu \mathrm{m}$. EGaIn will fill the electrode pseudochannels without leaking through the posts as long as the critical pressure to drive EGaIn into the channels is smaller than through the posts. For the L-shape electrode (B), we kept the distance between posts at $50 \mu \mathrm{m}$ and EGaIn still filled the electrode pseudochannels without leaking. Notably, the meniscus formed by EGaIn between PDMS posts in (B) has a smaller radius of curvature than (A), which can be explained by the increase in the critical pressure required to inject EGaIn 
through the electrode channel in (B) due to increased curvature of the channel; EGaIn was less resistive to flow through the posts. To fabricate EGaIn electrodes (C) and (D), water must first be injected into the channels to create a slip layer between EGaIn and PDMS that allows the liquid metal to flow smoothly instead of sticking to the surface of PDMS. ${ }^{54}$ However, the yields of (C) and (D) were significantly lower than their counterparts since we decreased the width of the channels to $160 \mu \mathrm{m}$ while keeping the distance between the posts at $50 \mu \mathrm{m}$. The slip layer is necessary to prevent the leakage of EGaIn through the posts because it lowers the critical pressure to drive EGaIn through the channel more than through the posts. The meniscus of central EGaIn electrode in (C) shows a larger radius of curvature than its counterparts in the side channels, indicating that a lower pressure is required to inject EGaIn into a channel with posts on both sides than on single side. Thus, one EGaIn pseudochannel can create an electrode that is coupled to two PSI/Au electrodes in a single step.

To study the mechanical and electrical stability of the EGaIn electrodes, we measured the conductance $G_{\text {meas }}$ of an EGaIn electrode versus the strain applied to the PDMS chip. We applied a maximum strain of $70 \%$ to measure in the elastic region of the PDMS chip (Figure S1) and a fixed bias of $0.1 \mathrm{~V}$ at the inlet of the microelectrode and grounded the outlet. We measured three types of EGaIn electrodes: (A) an EGaIn electrode defined in a pseudochannel separated by a single row of pillars of PDMS (Figure 5A, left); (B) an EGaIn electrode defined in a pseudochannel separated by rows of pillars of PDMS on either side of the electrode (Figure 5A, center); and (C) a reticulated EGaIn electrode defined in a serpentine pseudochannel separated by rows of pillars of PDMS on either side of the electrode (Figure 5A, right). Figure 5B shows that the three different types of electrodes return to their initial configuration after stretching (i.e., they do not leak).

We modeled the change in conductance $G_{\text {calc }}$ of the EGaIn anticipated due to the deformation of the electrode during stretching by treating it as a cuboid and calculating the conductance as a function of strain

$$
G_{\text {calc }}=\frac{1}{R}=\frac{A}{\rho \cdot L}=\frac{V}{\rho \cdot L^{2}}=\frac{V}{\rho \cdot L_{0}^{2} \cdot(e+1)^{2}}
$$

where, $R$ is the resistance, $\rho$ is the resistivity, $L$ is the length of the cuboid, $A$ is the cross-section of the cuboid, $V$ is the volume of the cuboid, $L_{0}$ is the original length of the cuboid, and $e$ is the strain of the cuboid. As long as $G_{\text {meas }} \geq G_{\text {calc }}$, the EGaIn electrodes are intact and are deforming with the PDMS chip without rupturing.

Figure 6 shows that the $G_{\text {meas }}$ is stable at low strain and drops sharply, approaching $G_{\text {calc }}$ around $25 \%$ strain and leveling off again around 60\% strain (see also Figure S12). Equation 1 suggests that in an ideal case, $G$ is proportional to $1 /(e+1)^{2}$, which disagrees with the experimental data as shown in Figure 6A. When the device is stretched, the crosssectional area of the microchannel is reduced, leading to an increasing shear stress (defined by the ratio between the force and the cross-section area parallel to the force vector) on the EGaIn electrode. Because of the unique shear-thinning properties of EGaIn, the electrode is resistive to deformation at a low shear stress, thus, the small change of conductance before the strain is at $25 \%$. As the shear stress continues to build, EGaIn yields and flows to the inlet and outlet of the microchannel, decreasing the cross-section area of the
(A)
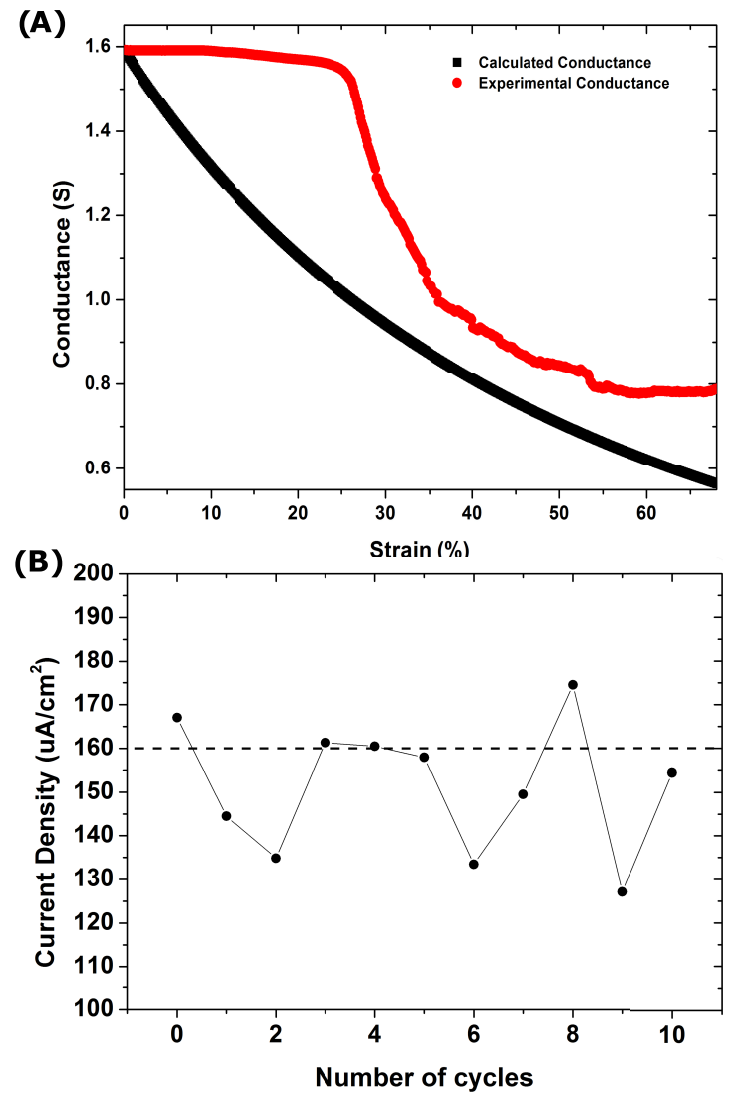

Figure 6. A) Plots of the experimental conductance $G_{\text {meas }}$ (red) and calculated conductance $G_{\text {calc }}$ (black) from eq 1 of EGaIn electrodes as a function of applied strain. (B) Evolution of the average photocurrent density of two devices fabricated from $\mathrm{Au}^{\mathrm{SE}}$ under 10 stretch/release cycles. The dashed line indicates the average photocurrent density before and after the cycles.

electrode. The plateau around $60 \%$ is likely due to nonuniform stretching of the PDMS (e.g., necking). Importantly, $G_{\text {meas }} \geq G_{\text {calc }}$ for all values of strain and $G_{\text {meas }}$ returns to its initial value when the strain is removed (Figure S10). To further test the stretchability of working devices, we measured the evolution of photocurrent densities of devices fabricated from $\mathrm{Au}^{\mathrm{SE}}$ electrodes under 10 stretch/release cycles. We applied a strain of $18 \%$ on the devices during each cycle and recorded their photoresponse after the cycle (Figure 6B). The tested devices produced stable photocurrents that fluctuate randomly instead of showing a clear trend of decay, which suggests that the integrity of the devices was preserved and the device is stretchable under a certain level of strain.

Regeneration. The reversible interaction between the charged surface residues of the PSI sub-units and the sulfonate moiety of the MPS linker not only facilitates the immobilization and orientation of the complexes, but also enables the exchange between the immobilized and the free PSIs (i.e., by mass action/self-assembly). Thus, exposure to a solution of active PSI should result in the recovery of $J_{S C}$ of an aged device as inactive PSI is displaced from the surface of the $\mathrm{Au}^{\mathrm{SE}}$ electrode and replaced with active PSI from solution. To test this hypothesis, we measured $J_{\mathrm{SC}}$ for three different bio-PV devices over 12 days. On day six, we circulated a solution of active PSI through the pseudochannel containing the redox couple and PSI $/ \mathrm{Au}^{\mathrm{SE}}$. Figure 7 shows that $J_{\mathrm{SC}}$ decreased in time for all three devices over the first three days, recovered to 


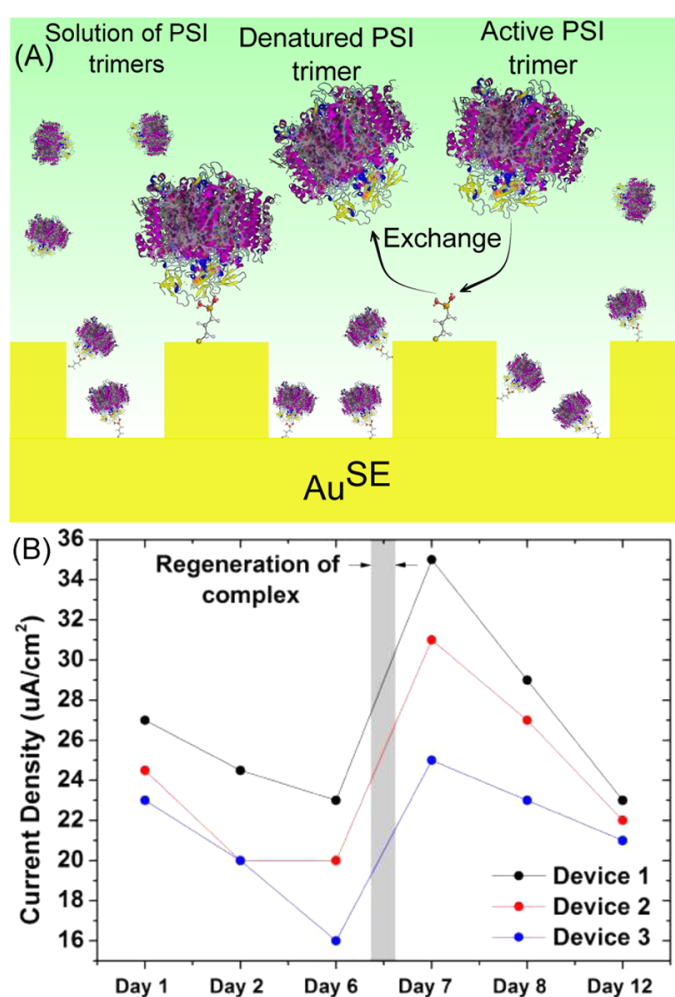

Figure 7. (A) Schematic illustration of the exchange between active and inactive PSI complexes on the surface of $\mathrm{Au}^{\mathrm{SE}}$. (B) Photocurrent of three devices measured over 12 days with an infusion of a solution of active PSI after day six.

values greater than or equal to the initial values and then continued to decrease over the subsequent three days. It is not clear why $J_{S C}$ increased above the initial values, but in no case did they exceed the maximum observed value for the asfabricated devices shown in Figure 2. To exclude the effect that the electrolyte was also replenished in the regeneration of PSI, we performed a control experiment in which only the electrolyte was replenished every day. Figure S5 shows a gradual decrease in photocurrent density in the presence of fresh electrolyte over 5 days, whose trend agrees with the observation in the previous experiment. By combining the results, we concluded that the denatured PSI is the contributor to the decay in the performance of the device.

To gain more insight into the effectiveness of the selfregeneration, we investigated the evolution of $V_{\mathrm{OC}}, J_{\mathrm{SC}}$, and FF before and after the infusion of active PSI (Figure S4 and Table S3). The $V_{\mathrm{OC}}$ does not show a significant decay during the measurement, however, the FF of the device decreases by $22 \%$ over six days before or after the exchange, which suggests that back electron transfer and recombination increase as the device degrades. Commensurate with $J_{\mathrm{SC}}$, the FF increased above the value at Day One after the infusion.

There are two plausible, simultaneous mechanisms for the degradation in the performance of the bio-PV devices over time; denaturation of the PSI units and dissociation of the PSI complexes from the surface. The drop in $J_{\mathrm{SC}}$ can be ascribed to either: it is a reflection of the decrease in the absolute number of active PSI complexes. The drop in the FF could be the result of partially denatured PSI sub-units with intact antenna complexes that can still absorb light, but cannot inject an electron into the $\mathrm{Au}^{\mathrm{SE}}$ electrode. The fact that the $V_{\mathrm{OC}}$ does not decrease is a strong indication that $J_{\mathrm{SC}}$ is always generated by PSI, a value that is determined by the electron transport chain in the reaction center of PSI and the redox potentials of the redox couples. When a typical PV device (e.g., Si p/n or organic/hybrid bulk-heterojunction) degrades, traps form, causing a drop in the $V_{\mathrm{OC}}$ because the energy levels are defined by a band structure. ${ }^{32}$ The stability of the $V_{\mathrm{OC}}$ is a reflection of the fact that, in effect, each PSI complex functions as a separate photovoltaic generator.

\section{CONCLUSIONS}

The leaves of plants are mechanically deformable structures that generate (chemical) energy from light and that selfregenerate via a circulatory system. Using a simple strategy of cofabrication and self-assembly, we have demonstrated soft, stretchable bio-PV that are capable of self-regeneration via an open circulatory system into which active PSI can be infusedapart from their appearance, these devices share key properties with leaves. The design of the reticulated electrode/ pseudochannel structure allows devices to be stacked to compensate for the relatively low optical cross-section, not entirely unlike the hierarchal stacking of thylakoids and chloroplasts.

Although bio-PV devices based on PSI are a long way from technological relevance, the fundamental appeal of co-opting proteins from the photosynthetic pathways of living organisms is the potential to incorporate the remarkable properties of photosynthetic organisms into artificial devices. Further study is needed to optimize the power conversion efficiency and better understand the mechanism of degeneration and selfregeneration, but we have demonstrated that PSI is the active component of our bio-PV devices and, therefore, that the recovery of degraded devices is due to the spontaneous replacement of inactive PSI by active PSI from solution.

\section{EXPERIMENTAL SECTION}

Cell Growth. The thermophilic cyanobacterium Thermosynechococcus elongatus BP-1 was grown under agitation (180 rpm) in BG11 medium with continuous $\mathrm{CO}_{2}$ supply at $1 \mathrm{~L} / \mathrm{min} .{ }^{55}$ The temperature was kept at $55{ }^{\circ} \mathrm{C}$, continuous light applied at 50-60 $\mu$ Einstein $\mathrm{m}^{-2}$ $\mathrm{s}^{-1}$ and cell growth pursued until late log phase. At last, the cells were harvested by centrifugation (JLA 9.100 rotor, Beckma; 5000g; 15 min) and resuspended in Buffer A (20 mM 4-(2-hydroxyethyl)-1piperazineethanesulfonic acid of $\mathrm{pH} 7.5 ; 10 \mathrm{mM} \mathrm{MgCl}_{2} ; 10 \mathrm{mM}$ $\mathrm{CaCl}_{2} ; 500 \mathrm{mM}$ Mannitol).

Thylakoid Membrane Preparation. Thylakoid membranes were prepared according to the following protocol, which represents a combination of two previously described preparation methods. ${ }^{56,57}$ To this end, fresh or frozen cells were resuspended in Buffer A and homogenized five times using a Dounce homogenizer. After the addition of lysozyme and DNase I (final concentrations: $5 \mathrm{mg} / \mathrm{mL}$ and $10 \mu \mathrm{g} / \mathrm{mL}$, respectively), the cell suspension was incubated under slow agitation for $1 \mathrm{~h}$ at $37{ }^{\circ} \mathrm{C}$ in the dark. Subsequently, the cells were lysed by three passages through a French Press (15000 psi; Constant Systems Limited, U.K.). Membranes were collected by centrifugation (JLA 16.250 rotor, Beckman; $38000 \mathrm{~g} ; 20 \mathrm{~min}$ ) and washed with Buffer A containing $3 \mathrm{M} \mathrm{NaBr}$. Afterwards, the membrane suspension was washed once with Buffer A and three times with a buffer containing $0.05 \% n$-dodecyl- $\beta$-D-maltoside (DDM) to remove the phycobilisomes. Finally, the thylakoid membranes were solubilized by incubation in Buffer A and supplemented with $0.6 \%$ DDM for $30 \mathrm{~min}$ at $20{ }^{\circ} \mathrm{C}$ in the dark. Nonsolubilized material was pelleted by centrifugation (JLA 16.250 rotor, Beckman; $38000 g$; 20 $\min$ ) and the supernatant was subjected to subsequent purification steps.

Photosystem I Purification. For PSI purification, fast liquid protein chromatography was applied on solubilized thylakoid 
membranes. The chromatographic purification was performed on a ÄKTA explorer (GE Healthcare) using an anion exchange column (HiTrap Q HP, GE Healthcare). After column equilibration with Buffer A $+0.03 \%$ DDM, the sample was applied and subsequently eluted by a linear gradient of $0-1 \mathrm{M} \mathrm{MgCl}_{2}$. The green fluorescent fractions were collected and desalted with Buffer A + 0.03\% DDM using Vivaspin 20 columns (molecular weight cut-off: $100 \mathrm{kDa}$; GE Healthcare). Finally, the purified PSI sample was adjusted to a Chl $a$ concentration of $300 \mu \mathrm{M}$ (with Buffer A + 0.03\% DDM), snap-frozen in liquid nitrogen and stored at $-80{ }^{\circ} \mathrm{C}$.

Determination of Chlorophyll $a$ and Protein Concentration. Chl $a$ determination was performed in $100 \%$ methanol as described by Porra et al. ${ }^{58}$

Fabrication of Microfluidic Channels. The fabrication of EGaIn microelectrodes begins with the generation of master patterns of microchannels on a silicon wafer using a negative photoresist (SU-8, Microchem). Curing PDMS over the patterns produces an inverse replica of the master and sealing it to a flat slab of PDMS defines the channels into which EGaIn is injected at a constant rate using a syringe pump. We designed the film mask (JD Photodata) for UV lithography on Clewin. We cleaned a 3 in. silicon wafer with acetone and isopropanol, and annealed it on a hotplate at $200{ }^{\circ} \mathrm{C}$ for $5 \mathrm{~min}$. After cooling the wafer to room temperature, we spin-coated $3 \mathrm{~mL}$ SU-8 photoresist on it in two stages: stage (1) with a velocity of 500 $\mathrm{rpm}$, acceleration of $100 \mathrm{rpm} / \mathrm{s}$ for $13 \mathrm{~s}$; stage (2) with a velocity of $2000 \mathrm{rpm}$, acceleration of $300 \mathrm{rpm} / \mathrm{s}$ for $45 \mathrm{~s}$. We then soft-baked the sample on a programmable hotplate in two stages: in stage (1) the temperature increased from room temperature to $95{ }^{\circ} \mathrm{C}$ in $35 \mathrm{~min}$ and maintained for $6 \mathrm{~min}$; in stage (2) the sample is allowed to slowly cool down to room temperature. We transferred the sample to the UV Mask Aligner for exposure. The mask was aligned with the sample to assure all patterns were projected onto the sample. We then exposed the sample to UV light (dosage $185-250 \mathrm{~mJ} / \mathrm{cm}^{2}$ ) for $34 \mathrm{~s}$. Post exposure bake was again carried out on a programmable hotplate in two stages: in stage (1) the temperature increased from room temperature to $95{ }^{\circ} \mathrm{C}$ in $75 \mathrm{~min}$ and maintained for $1 \mathrm{~min}$; in stage (2) the sample was allowed to slowly cool down to room temperature in a period of $6 \mathrm{~h}$. We developed the sample by immersing the sample into the developer (MR-Dev 600) on a glass Petri dish for $5 \mathrm{~min}$ and rinsed the sample with isopropanol. This procedure was cycled three times to assure the complete removal of the unexposed SU-8 photoresist and eventually the sample was dried in $\mathrm{N}_{2}$ to yield the master. We mixed the base and the curing agent (Sylgard 184 Silicone Elastomer) in a 10:1 (v/v) ratio by stirring and degassed the mixture in a desiccator for $30 \mathrm{~min}$. Then we carefully poured the mixture over the master in a plastic Petri dish and baked it at $60^{\circ} \mathrm{C}$ for $2 \mathrm{~h}$.

Fabrication of Shadow-Evaporated $\mathrm{Au}$ Electrode. We mounted the AAO membranes (Whatman Anodisc) against a rotating plate $(3 \mathrm{rpm})$. We centered the plate directly over the source and fixed the evaporation incident angle at $45^{\circ}$ to control the height of the nanotubes. After e-beam evaporating $150 \mathrm{~nm}$ of $\mathrm{Au}$ onto the membranes, we dissolved the alumina by immersing the coated membrane into $1 \mathrm{M} \mathrm{NaOH}$ (aq) for $1 \mathrm{~h}$. This procedure yielded an array of nanotubes supported by an electrically continuous backing of the same material.

Fabrication of Template-Stripped Au Electrode. We fabricated $\mathrm{Au}$ wires $(300 \mu \mathrm{m}$ wide, $100 \mathrm{~nm}$ thick) onto a 3 in. silicone wafer covered by a shadow mask using thermal deposition. Then, we silanized the wafer by putting it and a few droplets of trichloro $(1 \mathrm{H}, 1 \mathrm{H}, 2 \mathrm{H}, 2 \mathrm{H}$-perfluorooctyl)silane (Sigma-Aldrich, used as received) under vacuum for $30 \mathrm{~min}$. We glued clean glass substrates onto the Au wires using optical adhesive (Norland Optical Adhesive) cured in UV light. The glass was then mechanically cleaved, exposing an ultraflat Au surface.

Fabrication of Devices. To fabricate devices on shadowevaporated $\mathrm{Au}$ electrode, we started from carefully cutting a piece of AAO membrane $(15 \mathrm{~mm} \times 1 \mathrm{~mm})$ after the deposition of $\mathrm{Au}$, and placed it on a piece of PDMS substrate with the AAO side facing up. Then, we applied a few droplets of $1 \mathrm{M} \mathrm{NaOH}$ onto the membrane to remove the alumina in $1 \mathrm{~h}$. We rinsed the shadow-evaporated Au with deionized water and ethanol, and subsequently the electrode was dried in a continuous $\mathrm{N}_{2}$ flow. We first applied the aqueous solution of sodium 3-mercapto-1-propanesulfonate ( $1 \mathrm{mM}$, MPS, SigmaAldrich, used as received) to cover the whole electrode under the protection of a $\mathrm{N}_{2}$ atmosphere, allowing the formation of MPS SAM on the surface of $\mathrm{Au}$ in $2 \mathrm{~h}$. We then rinsed the sample with deionized water and immersed it in an aqueous solution of PSI $(1.04 \mu \mathrm{M})$ under the protection of a $\mathrm{N}_{2}$ atmosphere for $2 \mathrm{~h}$ to immobilize PSI onto the electrode. After rinsing the sample with deionized water and drying it under $\mathrm{N}_{2}$ flow, we encapsulated the Au electrode in one of the two microfluidic channels by manual alignment under an optical microscope. Then, we filled the other channel with EGaIn at a rate of $0.5 \mathrm{~mL} / \mathrm{h}$ to prevent any possible leakage between the PDMS posts due to high pressure while keeping the injection relatively fast. The channel of $\mathrm{Au}$ electrode was filled with an aqueous solution of ascorbic acid $(5 \mathrm{mM})$ and dichlorophenolindophenol (DCPIP, 0.1 $\mathrm{mM}$ ). Then, we sealed the inlet and outlet of the channel of the $\mathrm{Au}$ electrode with Kapton tape for measurement.

Measurement of Photocurrent. The device was allowed to cool down to $0{ }^{\circ} \mathrm{C}$ and remained at that temperature before the measurement. We connected the bottom Au electrode and the EGaIn electrode to a Keithley sourcemeter (model 6430 subfemtoamp remote sourcemeter), which can be controlled from a graphical user interface. The laser $(655 \mathrm{~nm}, 62 \mathrm{~mW})$ was fixed $9 \mathrm{~cm}$ above the surface of the device. For the measurement of light/dark cycles, the laser was on for $1 \mathrm{~min}$ and off for 4 min over 4 cycles in 20 min. For the measurement of $I-V$ characteristics, the device was measured in a bias window from -0.1 to $0.8 \mathrm{~V}(0 \rightarrow 0.8 \rightarrow 0 \rightarrow-0.1$ $\rightarrow 0 \mathrm{~V}$ ) with a step size of $0.01 \mathrm{~V}$.

Measurement of the Mechanical Properties. All devices were measured on Instron 5943 equipped with a $1 \mathrm{kN}$ load cell. The devices were held by two hydraulic grips and stretched at a constant rate of $3 \mathrm{~mm} / \mathrm{min}$ (strain rate $0.0016 / \mathrm{s}$ ). The gauge length between the two grips was measured by the software Bluehill 3 to interpret the Young's modulus of the devices. A bias of $0.1 \mathrm{~V}$ was applied between the outlet and inlet of the EGaIn microelectrodes through copper wires while the devices were being stretched. The currents were recorded by a Keithley 2400 instrument and recalculated into conductivity for better understanding. Three stretch/release cycle was performed on each EGaIn electrode with a strain magnitude of $70 \%$.

\section{ASSOCIATED CONTENT}

\section{Supporting Information}

The Supporting Information is available free of charge on the ACS Publications website at DOI: 10.1021/acsami.8b11115.

Young's modulus; control experiments; long-term stability; exchange of PSI; surface area estimations; atomic force microscopy data; scanning tunneling microscopy data; conductance vs strain data; power conversion efficiency calculations; energy-level diagrams (PDF)

\section{AUTHOR INFORMATION}

\section{Corresponding Author}

*E-mail: r.c.chiechi@rug.nl.

ORCID ${ }^{\circ}$

Hendrik W. de Vries: 0000-0002-9307-9015

Andreas Herrmann: 0000-0002-8886-0894

Ryan C. Chiechi: 0000-0002-0895-2095

\section{Present Addresses}

${ }^{\perp}$ Institute of Technical and Macromolecular Chemistry, RWTH Aachen University, Worringerweg 2, 52074 Aachen, Germany (A.H.).

"DWI-Leibniz Institute for Interactive Materials, Forckenbeckstr. 50, 52056 Aachen, Germany (A.H.). 


\section{Author Contributions}

${ }^{\S}$ X.Q. and O.C.O. contributed equally to this work.

Notes

The authors declare no competing financial interest.

\section{ACKNOWLEDGMENTS}

The Zernike Institute of Advanced Materials is gratefully acknowledged for financial support.

\section{REFERENCES}

(1) Mitchell Waldrop, M. The Chips Are Down for Moore's Law. Nature 2016, 530, 144-147.

(2) Service, R. F. The Brain Chip. Science 2014, 345, 614-616.

(3) Siegel, A. C.; Tang, S.; Nijhuis, C.; Hashimoto, M.; Phillips, S.; Dickey, M.; Whitesides, G. Cofabrication: A Strategy for Building Multicomponent Microsystems. Acc. Chem. Res. 2010, 43, 518-528.

(4) McDonald, J. C.; Whitesides, G. Poly(dimethylsiloxane) as a Material for Fabricating Microfluidic Devices. Acc. Chem. Res. 2002, 35, 491-499.

(5) Hung, L. H.; Lin, R.; Lee, A. Rapid Microfabrication of SolventResistant Biocompatible Microfluidic Devices. Lab Chip 2008, 8, 983-987.

(6) Becker, H.; Gartner, C. Polymer Microfabrication Methods for Microfluidic Analytical Applications. Electrophoresis 2000, 21, 12-26.

(7) Bustillo, J.; Howe, R.; Muller, R. Surface Micromachining for Microelectromechanical Systems. Proc. IEEE 1998, 86, 1552-1574.

(8) Mijatovic, D.; Eijkel, J.; van den Berg, A. Technologies for Nanofluidic Systems: Top-down vs Bottom-up-a Review. Lab Chip 2005, 5, 492-500.

(9) Kim, J.; et al. Epidermal Electronics with Advanced Capabilities in Near-Field Communication. Small 2015, 11, 906-912.

(10) Webb, R. C.; et al. Ultrathin Conformal Devices for Precise and Continuous Thermal Characterization of Human Skin. Nat. Mater. 2013, 12, 938-944.

(11) Liu, Y.; et al. Epidermal Mechano-Acoustic Sensing Electronics for Cardiovascular Diagnostics and Human-Machine Interfaces. Sci. Adv. 2016, 2, No. e1601185.

(12) Nagale, M.; Fritsch, I. Individually Addressable, Submicrometer Band Electrode Arrays. 1. Fabrication from Multilayered Materials. Anal. Chem. 1998, 70, 2902-2907.

(13) Ordeig, O.; Godino, N.; Campo, J.; Muñoz, F.; Nikolajeff, F.; Nyholm, L. On-Chip Electric Field Driven Electrochemical Detection Using a Poly(dimethylsiloxane) Microchannel with Gold Microband Electrodes. Anal. Chem. 2008, 80, 3622-3632.

(14) Gavin, P.; Ewing, A. Continuous Separations with Microfabricated Electrophoresis - Electrochemical Array Detection. J. Am. Chem. Soc. 1996, 118, 8932-8936.

(15) Choi, J. W.; Rosset, S.; Niklaus, M.; Adleman, J.; Shea, H.; Psaltis, D. 3-Dimensional Electrode Patterning within a Microfluidic Channel Using Metal Ion Implantation. Lab Chip 2010, 10, 783-788.

(16) Harnett, C. K.; Templeton, J.; Dunphy-Guzman, K.; Senousy, Y.; Kanouff, M. Model Based Design of a Microfluidic Mixer Driven by Induced Charge Electroosmosis. Lab Chip 2008, 8, 565-572.

(17) Liu, Y.; Pharr, M.; Salvatore, G. Lab-on-Skin: A Review of Flexible and Stretchable Electronics for Wearable Health Monitoring. ACS Nano 2017, 11, 9614-9635.

(18) Dickey, M.; Chiechi, R.; Larsen, R.; Weiss, E.; Weitz, D.; Whitesides, G. Eutectic Gallium-Indium (EGaIn): A Liquid Metal Alloy for the Formation of Stable Structures in Microchannels at Room Temperature. Adv. Funct. Mater. 2008, 18, 1097-1104.

(19) Kim, H.; Son, C.; Ziaie, B. A Multiaxial Stretchable Interconnect Using Liquid-Alloy-Filled Elastomeric Microchannels. Appl. Phys. Lett. 2008, 92, No. 011904.

(20) Cheng, S.; Rydberg, A.; Hjort, K.; Wu, Z. Liquid Metal Stretchable Unbalanced Loop Antenna. Appl. Phys. Lett. 2009, 94, 92-95.

(21) Gordiichuk, P. I.; Wetzelaer, G.; Rimmerman, D.; Gruszka, A.; De Vries, J.; Saller, M.; Gautier, D.; Catarci, S.; Pesce, D.; Richter, S.;
Blom, P.; Herrmann, A. Solid-State Biophotovoltaic Cells Containing Photosystem I. Adv. Mater. 2014, 26, 4863-4869.

(22) Carmeli, I.; Frolov, L.; Carmeli, C.; Richter, S. Photovoltaic Activity of Photosystem I-Based Self-Assembled Monolayer. J. Am. Chem. Soc 2007, 129, 12352-12353.

(23) Ciesielski, P.; Hijazi, F.; Scott, A.; Faulkner, C.; Beard, L.; Emmett, K.; Rosenthal, S.; Cliffel, D.; Kane Jennings, G. Photosystem I - Based Biohybrid Photoelectrochemical Cells. Bioresour. Technol. 2010, 101, 3047-3053.

(24) Gordiichuk, P.; Pesce, D.; Castañeda Ocampo, O.; Marcozzi, A.; Wetzelaer, G.; Paul, A.; Loznik, M.; Gloukhikh, E.; Richter, S.; Chiechi, R.; Herrmann, A. Orientation and Incorporation of Photosystem I in Bioelectronics Devices Enabled by Phage Display. Adv. Sci. 2017, 26, No. 1600393.

(25) Castañeda Ocampo, O.; Gordiichuk, P.; Catarci, S.; Gautier, D.; Herrmann, A.; Chiechi, R. Mechanism of Orientation-Dependent Asymmetric Charge Transport in Tunneling Junctions Comprising Photosystem I. J. Am. Chem. Soc. 2015, 137, 8419-8427.

(26) Mukhopadhyay, S.; Dutta, S.; Pecht, I.; Sheves, M.; Cahen, D. Conjugated Cofactor Enables Efficient Temperature-Independent Electronic Transport Across $6 \mathrm{~nm}$ Long Halorhodopsin. J. Am. Chem. Soc 2015, 137, 11226-11229.

(27) Kumar, K. S.; Pasula, R.; Lim, S.; Nijhuis, C. Long-Range Tunneling Processes across Ferritin-Based Junctions. Adv. Mater. 2016, 28, 1824-1830.

(28) Lee, I.; Lee, J.; Greenbaum, E. Biomolecular Electronics: Vectorial Arrays of Photosynthetic Reaction Centers. Phys. Rev. Lett. 1997, 79, 3294-3297.

(29) Mershin, A.; Matsumoto, K.; Kaiser, L.; Yu, D.; Vaughn, M.; Nazeeruddin, K.; Bruce, B.; Graetzel, M.; Zhang, S. Self-Assembled Photosystem-I Biophotovoltaics on Nanostructured $\mathrm{TiO}_{2}$ and $\mathrm{ZnO}$. Sci. Rep. 2012, 2, No. 234.

(30) Rizzo, A.; Cester, A.; Madsen, M.; Krebs, F.; Gevorgyan, S. A Novel Algorithm for Lifetime Extrapolation, Prediction, and Estimation of Emerging PV Technologies. Small Methods 2018, 2, No. 1700285 .

(31) Gevorgyan, S.; et al. Baselines for Lifetime of Organic Solar Cells. Adv. Energy Mater. 2016, 6, No. 1600910.

(32) Mateker, W.; McGehee, M. Progress in Understanding Degradation Mechanisms and Improving Stability in Organic Photovoltaics. Adv. Mater. 2017, 29, No. 1603940.

(33) Siegel, A.; Bruzewicz, D.; Weibel, D.; Whitesides, G. Microsolidics: Fabrication of Three-Dimensional Metallic Microstructures in Poly(dimethylsiloxane). Adv. Mater. 2007, 19, 727-733.

(34) Shevkoplyas, S. S.; Siegel, A.; Westervelt, R.; Prentiss, G.; Whitesides, G. The Force Acting on a Superparamagnetic Bead Due to an Applied Magnetic Field. Lab Chip 2007, 7, 1294-1302.

(35) Psaltis, D.; Quake, S.; Yang, C. Developing Optofluidic Technology through the Fusion of Microfluidics and Optics. Nature 2006, 442, 381-386.

(36) Wolfe, D. B.; Conroy, R.; Garstecki, P.; Mayers, B.; Fischbach, M.; Paul, K.; Prentiss, M.; Whitesides, G. Dynamic Control of LiquidCore Liquid-Cladding Optical Waveguides. Proc. Natl. Acad. Sci. U. S. A. 2004, 101, 12434-12438.

(37) Tang, S. K.; Stan, C.; Whitesides, G. Dynamically Reconfigurable Liquid-Core Liquid-Cladding Lens in a Microfluidic Channel. Lab Chip 2008, 8, 395-401.

(38) Mayers, B. T.; Vezenov, D.; Vullev, V.; Whitesides, G. Arrays and Cascades of Fluorescent Liquid - Liquid Waveguides: Broadband Light Sources for Spectroscopy in Microchannels. Anal. Chem. 2005, 77, 1310-1316.

(39) Tang, S. K.; Li, Z.; Abate, A.; Agresti, J.; Weitz, D.; Psaltis, D.; Whitesides, G. A Multi- Color Fast-Switching Microfluidic Droplet Dye Laser. Lab Chip 2009, 9, 2767-2771.

(40) Garstecki, P.; Ganan-Calvo, A.; Whitesides, G. Formation of Bubbles and Droplets in Microfluidic Systems. Bull. Pol. Acad. Sci. 2005, 53, 361-372.

(41) Shah, R.; Shum, H.; Rowat, A.; Lee, D.; Agresti, J.; Utada, A.; Chu, L.; Kim, J.; Fernandez-Nieves, A.; Martinez, C.; Weitz, D. 
Designer Emulsions Using Microfluidics. Mater. Today 2008, 11, 1827.

(42) Thorsen, T.; Roberts, R.; Arnold, F.; Quake, S. Dynamic Pattern Formation in a Vesicle-Generating Microfluidic Device. Phys. Rev. Lett. 2001, 86, 4163-4166.

(43) Xu, S.; Nie, Z.; Seo, M.; Lewis, P.; Kumacheva, E.; Stone, H.; Garstecki, P.; Weibel, D.; Gitlin, I.; Whitesides, G. Generation of Monodisperse Particles by Using Microfluidics: Control over Size, Shape, and Composition. Angew. Chem., Int. Ed. 2005, 44, 724-728. (44) Garstecki, P.; Gitlin, I.; Diluzio, W.; Whitesides, G.; Kumacheva, E.; Stone, H. Formation of Monodisperse Bubbles in a Microfluidic Flow-Focusing Device. Appl. Phys. Lett. 2004, 85, 26492651.

(45) Zheng, B.; Roach, L.; Ismagilov, R. Screening of Protein Crystallization Conditions on a Microfluidic Chip Using NanoliterSize Droplets. J. Am. Chem. Soc. 2003, 125, 11170-11171.

(46) Song, H.; Chen, D.; Ismagilov, R. Reactions in Droplets in Microfluidic Channels. Angew. Chem., Int. Ed. 2006, 45, 7336-7356.

(47) O’Regan, B.; Gratzel, M. A Low-Cost, High-Efficiency SolarCell Based on Dye-Sensitized Colloidal $\mathrm{TiO}_{2}$ Films. Nature 1991, 353, 737-740.

(48) So, J.-U.; Dickey, M. Inherently Aligned Microfluidic Electrodes Composed of Liquid Metal. Lab Chip 2011, 11, 905-911.

(49) Weiss, E. A.; Kaufman, G.; Kriebel, J.; Li, Z.; Schalek, R.; Whitesides, G. $\mathrm{Si} / \mathrm{SiO}_{2}$-Templated Formation of Ultraflat Metal Surfaces on Glass, Polymer, and Solder Supports: Their Use as Substrates for Self-Assembled Monolayers. Langmuir 2007, 23, 96869694.

(50) Allen, J.; Holmes, N. In Photosynthesis: Energy Transduction: A Practical Approach; Hipkins, M., Baker, N., Eds.; IRL Press: Oxford, 1986; Chapter 5, pp 103-141.

(51) Dickey, M. D.; Weiss, E.; Smythe, E.; Chiechi, R.; Capasso, F.; Whitesides, G. Fabrication of Arrays of Metal and Metal Oxide Nanotubes by Shadow Evaporation. ACS Nano 2008, 2, 800-808.

(52) Ciesielski, P.; Faulkner, C.; Irwin, M.; Gregory, J.; Tolk, N.; Cliffel, D.; Jennings, G. Enhanced Photocurrent Production by Photosystem I Multilayer Assemblies. Adv. Funct. Mater. 2010, 20, 4048-4054.

(53) Ciesielski, P. N.; Scott, A.; Faulkner, C.; Berron, B.; Cliffel, D.; Kane Jennings, G. Functionalized Nanoporous Gold Leaf Electrode Films for the Immobilization of Photosystem I. ACS Nano 2008, 2, 2465-2472.

(54) Khan, M.; Trlica, C.; So, J.; Valeri, M.; Dickey, M. Influence of Water on the Interfacial Behavior of Gallium Liquid Metal Alloys. ACS Appl. Mater. Interfaces 2014, 6, 22467-22473.

(55) Rippka, R.; Deruelles, J.; Waterbury, J.; Herdman, M.; Stanier, R. Generic Assignments, Strain Histories and Properties of Pure Cultures of Cyanobacteria. Microbiology 1979, 111, 1-61.

(56) El-Mohsnawy, E.; Kopczak, M.; Schlodder, E.; Nowaczyk, M.; Meyer, H.; Warscheid, B.; Karapetyan, N. V.; Rögner, M. Structure and Function of Intact Photosystem 1 Monomers from the Cyanobacterium Thermosynechococcus elongatus. Biochemistry 2010, 49, 4740-4751.

(57) Mukherjee, D.; May, M.; Vaughn, M.; Bruce, B.; Khomami, B. Controlling the Morphology of Photosystem I Assembly on ThiolActivated Au Substrates. Langmuir 2010, 26, 16048-16054.

(58) Porra, R.; Thompson, W.; Kriedemann, P. Determination of Accurate Extinction Coefficients and Simultaneous Equations for Assaying Chlorophylls a and b Extracted with Four Different Solvents: Verification of the Concentration of Chlorophyll Standards by Atomic Absorption Spectroscopy. Biochim. Biophys. Acta, Bioenerg. 1989, 975, 384-394. 